\title{
Performance Evaluation of OFDM Transceiver For BPSK \& QAM Modulation Techniques
}

\author{
Dipa Nitin Kokane ${ }^{1,}$ Nitin H kokane ${ }^{2}$ \\ ${ }^{1}$ (Sr. Asst. Prof, ABES Engg College, \\ ${ }^{2}$ (Manager, Bharat Electronics Ltd,
}

\begin{abstract}
The telecommunication industries insisted on new technology with high transmission abilities standardized by IEEE 802.16 also referred as WiMAX .It has two patterns fixed(802.16d-2004) and mobile(802.16d-2005) which are based on OFDM. This paper is about study and implementation of Convolutional coded WiMAX system comparing different modulation schemes such as BPSK and QAM. The performance has been concluded based on BER and SNR through MATLAB Simulation. Keywords-BER, BPSK, IFFT, IEEE 802.16 OFDM, QAM, SNR, WiMAX
\end{abstract}

\section{INTRODUCTION}

WiMAX is introduced by the Institute of Electrical and Electronic Engineers(IEEE) which is designated by 802.16 to provide world wide interoperability for microwave access. There are fixed(802.16d) and mobile(802.16e) WiMAX.This technology offers a high speed,secure,sophisticate, last mile broadband service,ensuring a flexible and cheap solution to certain rural access zones.In a fixed wireless communication, WiMAX can replace the telephone company's copper wire networks, the cable TV's coaxial cable infrastructure. In its mobile variant it can replace cellular networks. In comparison with Wi-Fi and Cellular technology, Wi- Fi provides a high data rate, but only on a short range of distances and with a slow movement of the user. And Cellular offers larger ranges and vehicular mobility, but it provides lower data rates, and requires high investments for its deployment. WiMAX tries to balance this situation. WiMAX fills the gap between Wi-Fi and Cellular, thus providing vehicular mobility, and high service areas and data rates .WiMAX is a standards based technology for wireless MANs conforming to parameters which enable interoperability. WiMAX developments have been rapidly moving forward. since the initial standardization efforts in IEEE 802.16. Standards for Fixed WiMAX (IEEE 802.16d- 2004) were announced as final in 2004, followed by Mobile WiMAX (IEEE 802.16e) in 2005, which are based on orthogonal frequency division multiplexing (OFDM) technology. OFDM is a transmission technique built for high speed bi-directional wired or wireless data communication[1,2].OFDM has high PAPR.

\section{Advantages of OFDM :}

1. Channel bandwidth is divided into multiple subchannels ,i.e multicarrier transmission which is a method for the efficient utilization of the band width.

2. Subcarriers are orthogonal to each other in frequency domain which reduces ISI and frequency- selective fading. The technique is based upon the idea of multi-carrier modulation (MCM) where transmitted data is modulated on several orthogonal carrier frequencies. The subcarriers are closely spaced together but still orthogonal, which means that they are perpendicular in a mathematical sense, and do not interfere with each other.

The implementations of OFDM in WIMAX technology today have been done by using FFT and its inverse operation IFFT,uses the formula given below.[1]

$$
x_{n}=\left(\frac{1}{N}\right) \sum_{k=0}^{N-1} X_{k} e^{2 \pi \cdot j n k / N} ; \text { where } \mathrm{N}=0,1,2 \ldots \mathrm{N}-1 .
$$

\section{SYSTEM INTEGRATION AND IMPLEMENTATION OF WORK FLOW}

In the development and testing of IEEE 802.16d Wireless MAN-OFDM PHY, the specifications of communication transfer have varying systems, which are based on our needs. For our study, we used the standard communication system box with a map provided by Matlab, which contains the following: Internal Communications Block set, Signal processing Block set, and Simulink Blockset. These correspond to our use of the hardware development platform. The overall WIMAX PHY system construction is opened in the Simulink 
interface and Matlab is used to communicate the internal functions. We intend to build a finished system into a module, in accordance with the code of each block. Through this, we can perform the compilation and completion that will be automatically compiled in Matlab. The overall system workflow is given below.

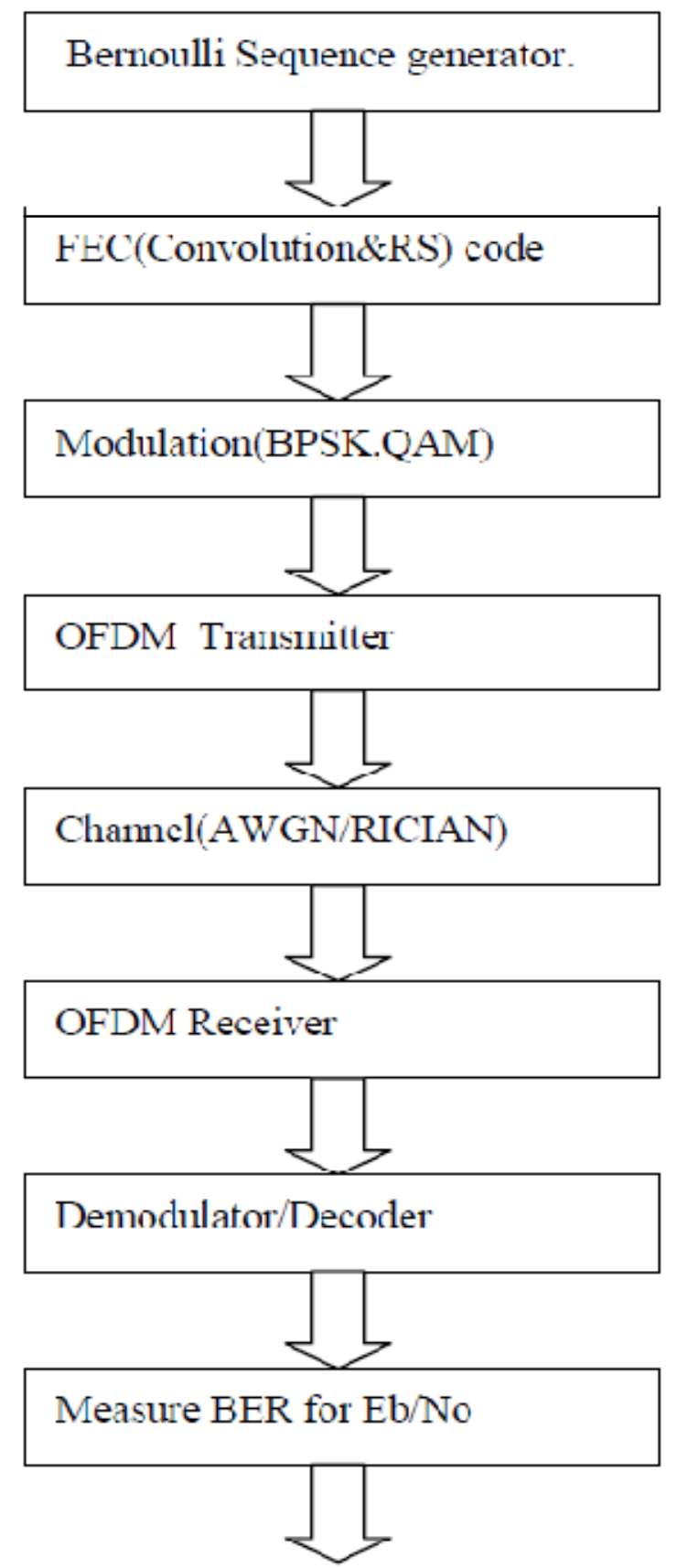

The basic block of wireless communication is given below fig. 1

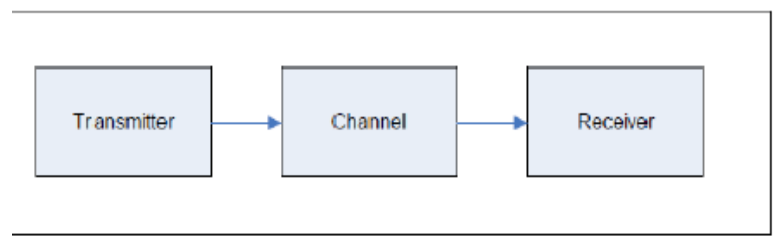

Fig.1 


\section{Where}

Transmitter block consists of:

1.Input is Bernoulli Binary

2.FEC(Convolution Code, RS Code)

3.Modulation(BPSK and QAM)

4.OFDM Transmitter(IFFT)

Channel can be AWGN,RICIAN and Both. And Receiver block consists of:

1.OFDM Receiver(FFT)

2.Demodulator

3.Decoder.(Viterbi decoder,RS Decoder)

Parameters for Simulation: Channel Bandwidth is 3.5MHz Cyclic Prefix-1/8

Output Datatype-Boolean

Samples per frame-864

The model for convolutional coded WiMAX system using BPSK modulation and QAM modulation is shown.

The detailed diagram is given below:

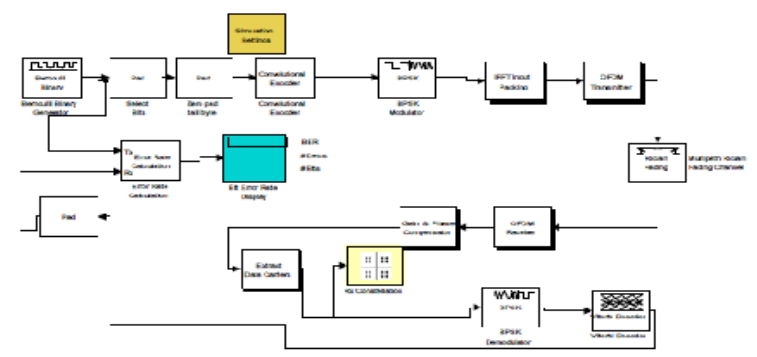

Fig. 2

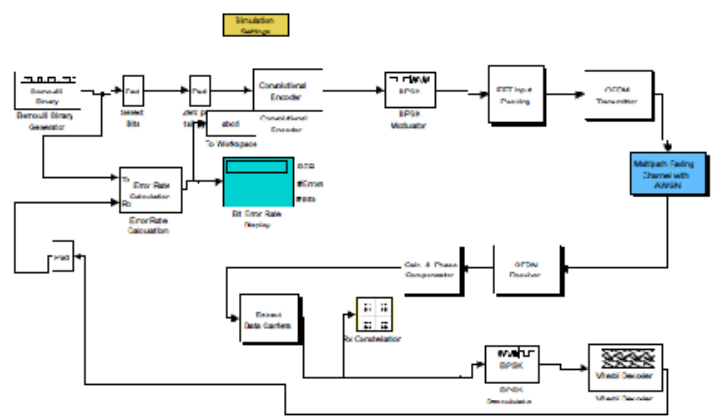

Tig.3

The above models are simulated in matlab the simulation rate is .025 and the results are recorded.

1.WITH OUT CHANNEL(QAM) fig.4



Fig 4 
2.WITH AWGN CHANNEL(QAM) fig 5

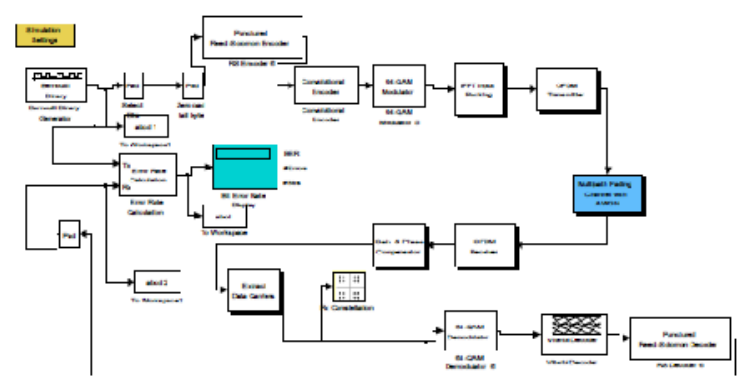

After constellation is observed as below fig.6

Fig 5



Fig 6

The output is observed for BPSK and QAM (fig.7, 8)

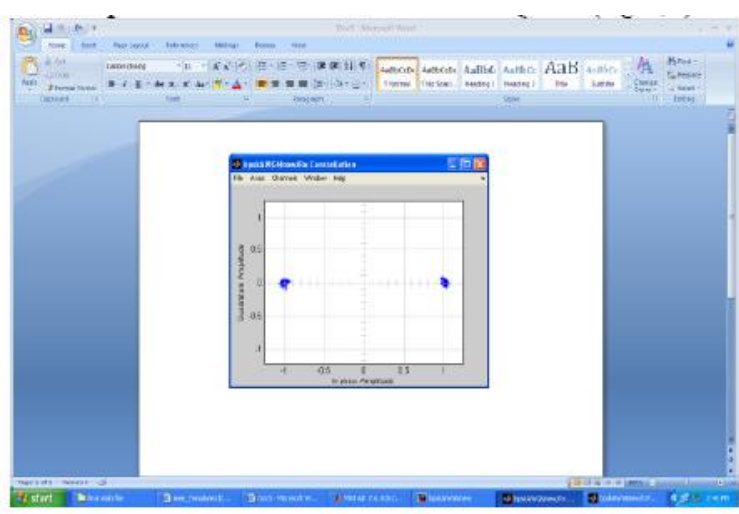

Fig 7

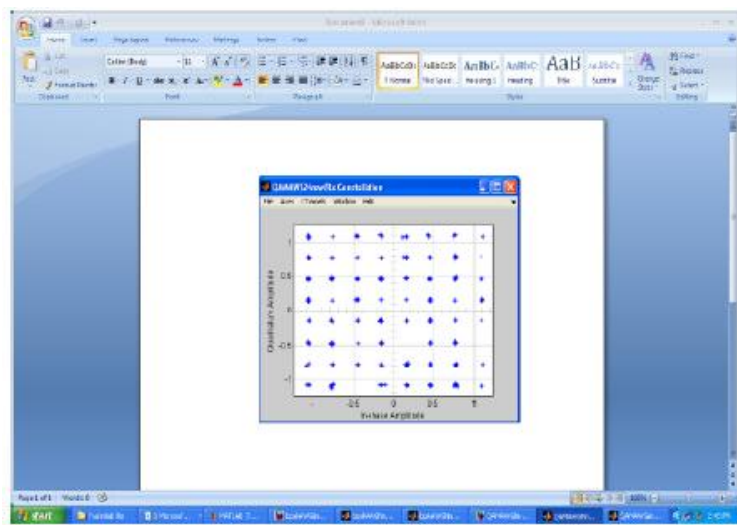

Fig 8 
The BER Figure is given below: Without channel

BER for bpsk :4471

BER for QAM : 00463

Through channel AWGN

BER for bpsk :4481

BER for QAM : 08141

Graph is in between different SNR vs BER. For BPSK Modulation Tech.

\begin{tabular}{|r|r|}
\hline SNR & \multicolumn{2}{l|}{ BER } \\
\hline 10 & 0.456 \\
\hline 20 & 0.4484 \\
\hline 30 & 0.448 \\
\hline 40 & 0.448 \\
\hline
\end{tabular}

Table1

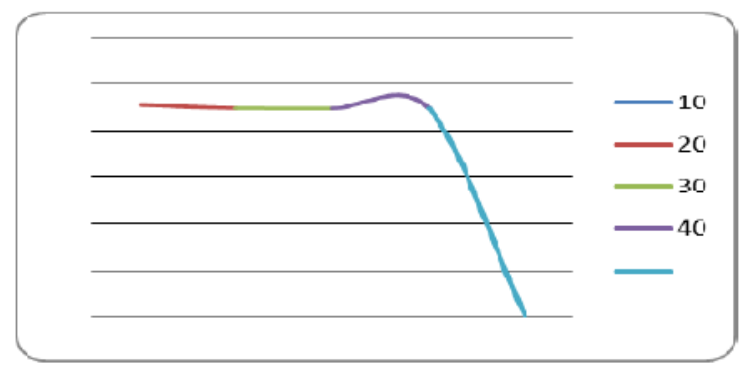

Graph 1

QAM Modulation Technique.

\begin{tabular}{|r|r|}
\hline SNR & BER \\
\hline 10 & 0.5 \\
\hline 20 & 0.4226 \\
\hline 30 & 0.08141 \\
\hline 40 & 0.01085 \\
\hline 50 & 0.005943 \\
\hline
\end{tabular}

Table 2

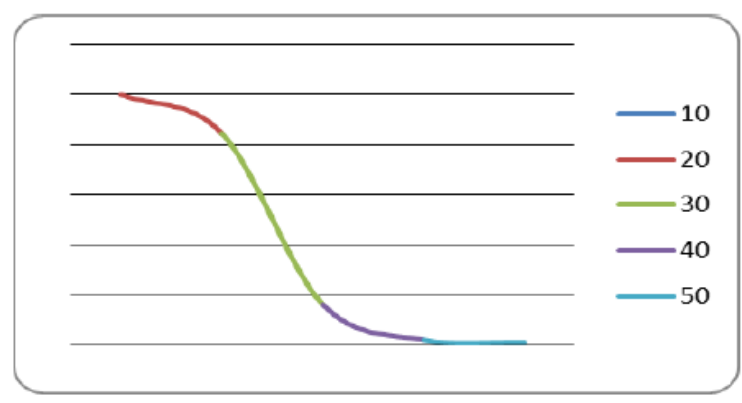

Graph 2 


\section{CONCLUSIONS}

It is observed that in convolutional coded BPSK system the BER-0.448, But it get reduced to ZERO Approximately in convolutional coded QAM system(BER- 0.00463).For further working on the system and sending through AWGN Channel we observed that the BER is less in case of QAM .

\section{REFERENCES}

[1] Intel (2006). Orthogonal Frequency Division Multiplexing. $<$ http://www.intel.com/netcomms/techn ologie s/wimax/303787.pdf>.

[2] Wikipedia (2006). <http://en.wikipedia.org/wiki/ OFDM>.

[3] WiMAX Forum (2006). http://www.wimaxforum. org/>.

[4] Intel (2006). Understanding Wi-Fi and WiMAX. <http://www.intel.com/netcomms/ technologies/wimax/ 304471.pdf>.

[5] WiMAX Forum (2004). WiMAX.stechnology for LOS and NLOS environments. <http://www.wimaxforum.org/ news/downloads/WiM AXN LOSgeneralversionaug04.pdf>.

[6] IEEE Standard 802.16-2004 (2004). <http://standards. ieee.org/getieee802/download/802.16-2004.pdf>.

[7] Michael Komara (2004). SDR Architecture Ideally Suited For Evolving 802.16 WiMAX Standards. <http://www.aircom.com/KomaraWiMAX.pdf>.

[8] J. Heiskala \& J. Terry (2001). OFDM Wireless LANs: A Theoretical and Practical Guide. Sams, 1st edition. ISBN 0672321572. 